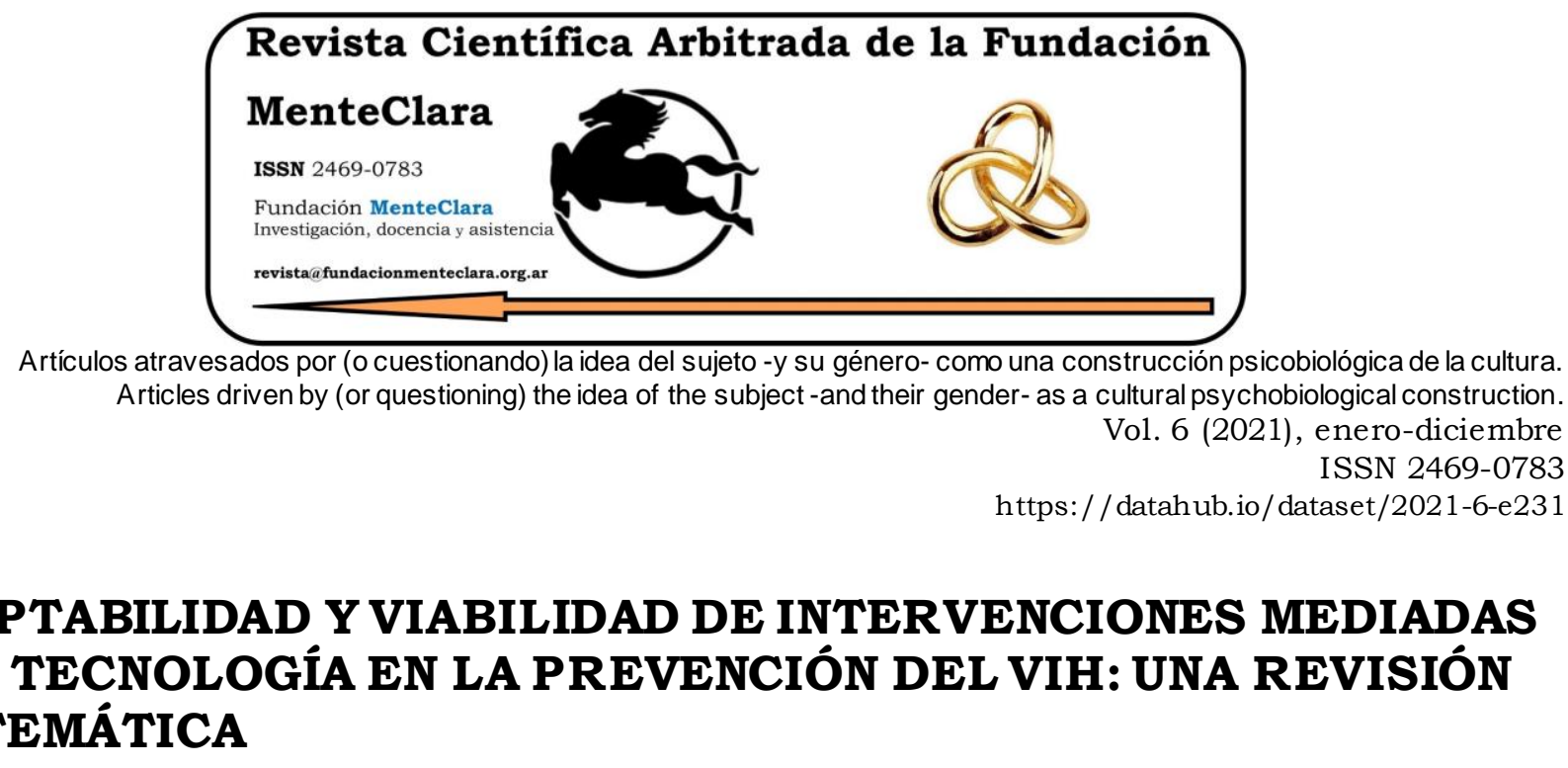

\title{
ACEPTABILIDAD Y VIABILIDAD DE INTERVENCIONES MEDIADAS POR TECNOLOGÍA EN LA PREVENCIÓN DEL VIH: UNA REVISIÓN SISTEMÁTICA
}

\author{
ACCEPTABILITY AND FEASIBILITY OF TECHNOLOGY-MEDIATED INTERVENTIONS \\ IN HIV PREVENTION: A SYSTEMATIC REVIEW
}

Ricardo Sánchez Medina ${ }^{1}$, Consuelo Rubi Rosales Piña ${ }^{2}$, Analía Verónica Losada ${ }^{3}$

Cómo citar este artículo / Citation: Sánchez-Medina, R., Rosales-Piña, C. R. \& Losada, A. V. (2021). Ace ptabilidad y viabilidad de intervenciones mediadas por tecnologia en la prevención del VIH: Una revisión sistemática. Revista Científica Arbitrada de la Fundación MenteClara, Vol. 6 (231). DOI: https://doi.org/10.32351/rca.v6.231

Copyright: (C) 2021 RCAFMC. Este artículo de acce so abierto es distribuido bajo los términos de la licencia Creative Commons Attribution 4.0 International License (CC BY 4.0). Recibido: 09/07/2021. Aceptado: 12/07/2021 Publicación online: 13/07/2021

Conflicto de intereses: Ninguno que declarar.

\section{Resumen}

El objetivo del presente trabajo fue analizar las publicaciones entre 2018 y 2020 sobre la aceptabilidad y viabilidad de intervenciones mediadas por tecnología orientadas en la prevención del VIH, dado que es la antesala de la evaluación de su efectividad, de tal manera que estos indicadores permitirán obtener mejores resultados en esta última fase y por lo cual es fundamental hacer esta valoración. A través de una revisión sistemática con el método PRISMA, se encontró un total de 17 artículos en tres bases de datos especializadas (EBSCO, Web of Science y Scopus). Se observa que la mayoría de las intervenciones son mediadas por internet para reducir conductas sexuales de riesgo, siendo dos estrategias las más utilizadas, entrevistas abiertas e

\footnotetext{
1 Universidad Nacional Autónoma de México, Facultad de Estudios Superiores Iztacala, México.

2 Universidad Nacional Autónoma de México, Facultad de Estudios Superiores Iztacala, México.

3 Universidad de Flores, Facultad de Psicología y Ciencias Sociales, Argentina.
} 
instrumentos en formato tipo Likert, además en todos los resultados de las investigaciones se encuentra que es factible y pertinente el uso de medios tecnológicos en las intervenciones para prevenir el VIH y se obtienen los resultados esperados; se discute la importancia de tomar en cuenta ambos indicadores para la evaluación de la aceptabilidad y viabilidad de las intervenciones, así como de la importancia de llevar a cabo esta valoración antes de llevar a cabo la intervención a gran escala.

\section{Abstract}

The objective of this study was to analyse publications from 2018 through 2020 on the acceptability and viability of technology-mediated HIV prevention interventions, taken as a leading indicator in evaluating their effectiveness, in such a way for these indicators to allow the acquisition of more accurate results in the final phase. Through a systematic review using the PRISMA method, a total of 17 papers were found in three specialized databases (EBSCO, Web of Science and Scopus). A majority of these interventions were mediated online to reduce risky sexual behaviors, with two strategies showing clear prevalence: Open interviews and questionnaires employing the Likert scale or analogous tools. The results of these investigations were unanimous in finding the use of technological means in preventing HIV both feasible and pertinent, meeting expected results. The importance of considering both indicators in evaluating the viability of these methods is discussed, as is the importance of carrying out this assessment prior to continuing such interventions on a larger scale.

Palabras Claves: intervención basada en internet; prevención del VIH; aceptabilidad; viabilidad

Keyw ords: internet-based intervention; HIV prevention; acceptability; viability 


\section{Introducción}

De acuerdo con el Programa Conjunto de las Naciones Unidas sobre el VIH/SIDA (ONUSIDA, 2021) a nivel mundial se tienen contabilizadas alrededor de 37.6 millones de personas que viven con VIH y tan solo en 2020 cerca de 1.5 millones contrajeron la infección por VIH y 690 mil personas fallecieron por enfermedades relacionadas con el SIDA, mostrando evidencia de la importancia que tiene continuar con programas orientados a reducir nuevas infecciones o reinfecciones por VIH.

Con base en lo anterior, se han diseño diversos programas de intervención orientados a diversos grupos que por el contexto en el que se encuentran son más susceptibles de infección por VIH, en los cuales se resalta la importancia del uso del condón en relaciones sexuales (Anstee et al., 2019) (Evans et al., 2020). Además en los últimos años, ha cobrado una gran relevancia las intervenciones a través del uso de la tecnología que busca tener una mayor cobertura y atender a población de dificil acceso (Mustanski et al., 2018) (Suryavanshi et al., 2020).

Si bien existe evidencia de la efectividad de intervenciones para promover la salud sexual a través de la tecnologia, tal y como lo muestra la revisión sistemática de Knight et al. (2017); es importante resaltar que esta avanza rápidamente, por lo cual es necesario que estas intervenciones se adapten a ello, así como a las necesidades y características de los usuarios, y que las intervenciones logren un cambio en el comportamiento sexual de las personas para reducir la infección por $\mathrm{VIH}$; lo anterior implica un arduo trabajo que propicia la publicación de resultados de manera paulatina; por ejemplo, desde la publicación del protocolo de intervención (Fan et al., 2020), la evaluación de 
aceptabilidad de la intervención (Bauermeister et al., 2019), o propiamente la efectividad de la intervención (Hightow-Weidman, 2019).

Lo anterior responde a lo que Fernández-Sánchez et al. (2020) señalan respecto a que toda intervención pasa por varias fases, desde el diseño de la intervención -fase 1-, la realización de un estudio piloto -fase 2- y la evaluación de su efectividad-fase 3-, es precisamente sobre la fase 2 en la que se centra el presente trabajo, debido a que una fase muy importante y que en muchas ocasiones no se le da la importancia y relevancia que merece, ya que es precisamente en esta fase en la que se tiene la oportunidad de aplicar la intervención a menor escala con el objetivo de valorar y mejorar la intervención, en términos de la aceptabilidad -ej. satisfacción de la intervención-, viabilidad -ej. es posible llevarla a cabo-, utilidad -ej. los usuarios consideran que lo aprendido lo pueden usar en su vida cotidiana-y usabilidad -ej. es fácil de usar el medio tecnológico propuesto en la intervención-.

En esta línea, se ha observado la importancia que tiene conocer estos elementos y de cómo esta información coadyuva en su mejora para una aplicación a mayor escala (Refugio et al., 2019) (Sullivan et al., 2017); sin embargo, se ha detectado que la forma en cómo se hace es diferente, desde quienes utilizar instrumentos en escala Likert (Gannon et al., 2020), hasta quienes hacen entrevistas abiertas (Dietrich et al., 2020), lo anterior es un indicador de cómo la información que se obtiene es diversa y posiblemente diferente, por lo que es necesario identificar qué y cómo se ha hecho para evaluar las intervenciones mediadas por tecnología para reducir la infección por VIH que permita tener un panorama general que coadyuve en la toma de decisiones respecto a qué medios tecnológicos se están utilizando en los últimos años, cuáles son sus objetivos y cómo se está evaluando la aceptabilidad y viabilidad de las intervenciones. 
Con base en lo anterior, el objetivo de la presente investigación es analizar las publicaciones realizadas entre 2018 y 2020, a través de una revisión sistemática, sobre la evaluación de la aceptabilidad y viabilidad de intervenciones mediadas por tecnología orientadas en la prevención del VIH.

\section{Materiales y métodos}

Revisión de la literatura

Se realizó una búsqueda de artículos científicos en tres bases de datos Ebsco, Web of Science y Scopus, que se caracterizan por incluir revistas que cumplen con un proceso riguroso de arbitraje y con una gran diversidad de revistas especializadas de todo el mundo, permitiendo tener un panorama global del trabajo académico. Los términos de búsqueda fueron en inglés y se utilizaron los operadores booleanos $A N D$ y $O R$, en cada base de datos se emplearon tres palabras clave y su combinación, intervention, HIV, technology, internet; los términos de búsqueda fueron consultados en las categorias Title, Abstract y Keywords.

Criterios de inclusión y exclusión

Los criterios de inclusión de artículos fueron publicaciones comprendidas en el periodo de 2018 a 2020. Artículos de revista, con acceso a texto completo, y en la que se describiera alguna intervención mediada por tecnología orientada en la prevención del VIH que evaluará la aceptabilidad o viabilidad por parte de los participantes. Como criterios de exclusión, se consideró la falta de consenso de los autores sobre la calidad metodológica de los estudios. 
Evaluación de la calidad metodológica

Se tomó en cuenta 12 de los 18 indicadores de la escala de calidad SQUIRE Guidelines 2.0 de Ogrinc et al., (2015) que corresponden al título, resumen, descripción del problema, objetivos, intervención, medidas, análisis, consideraciones éticas, resultados, interpretación, limitaciones y conclusiones. Un punto importante por resaltar es que, si bien se espera que dentro de la calidad metodológica en intervenciones es la presencia de al menos un grupo control, en la presente investigación solo bastó con que se incluyera el grupo experimental dado que en la evaluación de viabilidad y aceptabilidad generalmente son estudios piloto. Por otro lado, también fue de suma importancia que explicitarán los aspectos éticos, de tal forma que se garantice la seguridad e integridad de las personas en cada una de las intervenciones incluidas en la revisión.

\section{Procedimiento}

La revisión sistemática se basó en la declaración PRISMA-NMA (Hutton et al., 2016). A principios de 2021 se realizó una búsqueda de información en bases de datos especializadas, la primera se realizó en Ebsco, después Web of Science y por último, Scopus. Los primeros resultados arrojaron un total de 434 artículos publicados entre 2018 y 2020, en el cribado de registros se obtuvieron un total de 100 artículos que eran artículos de revista, con acceso al texto completo y que en el título, resumen o palabras clave estuvieran presentes los términos de búsqueda. Posteriormente, se realizó un análisis considerando los criterios de inclusión y exclusión, centrando el análisis sobre la implementación de una intervención mediada por tecnología en la prevención de VIH y en la que se señalara la evaluación de la aceptabilidad o viabilidad, quedando un total de 79 artículos. Todos estos 
artículos fueron gestionados en el programa EndNote X9, y posteriormente con base en la escala de calidad QUIRE Guidelines 2.0 (Ogrinc et al., 2015), los autores revisaron de manera individual los artículos para determinar la calidad de los artículos. Con base en lo anterior, se compararon los resultados de inclusión o exclusión, si había consenso se introducía o descartaba de la revisión sistemática; pero si la evaluación difería, entonces se discutía el artículo para delimitar su inclusión o exclusión, finalmente se eligieron 17 artículos -ver figura 1-.

\section{Figura 1}

Diagrama de flujo para la selección de artículos sobre la aceptabilidad o viabilidad de intervenciones mediadas por tecnología en la prevención del VIH.

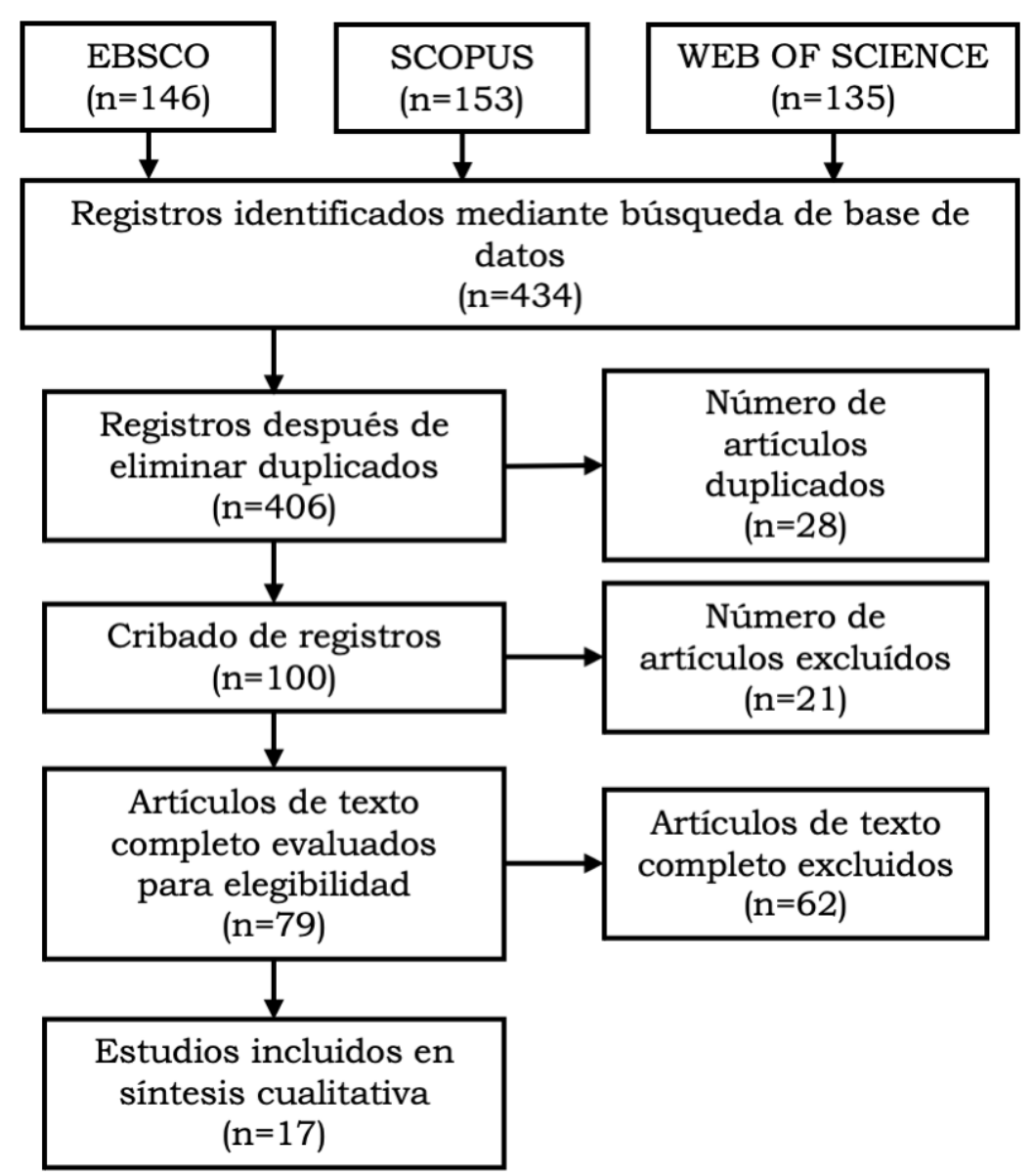




\section{Resultados}

En la Tabla 1 se presentan los principales resultados de 17 investigaciones publicadas durante 2018 a 2020 que evalúan la aceptabilidad y viabilidad de intervenciones mediadas por tecnología en la prevención del VIH.

\section{Tabla 1}

Estudios de evaluación sobre aceptabilidad $y$ viabilidad de intervenciones mediadas por tecnología en la prevención del VIH de 2018

a 2020.

\begin{tabular}{|c|c|c|c|c|c|}
\hline & Estudio & Muestra & In tervención & $\begin{array}{l}\text { Evaluación de } \\
\text { aceptabilidad y } \\
\text { viabilidad }\end{array}$ & Principales resultados \\
\hline $\mathbf{1}$ & $\begin{array}{l}\text { Arya et al. } \\
(2019)\end{array}$ & $\begin{array}{l}53 \text { médicos } \\
\text { de diferentes } \\
\text { clinicas. } \\
\text { Edad } \\
\text { promedio: } 33 \\
\text { años. }\end{array}$ & $\begin{array}{l}\text { Video para } \\
\text { promover pruebas de } \\
\text { detección del VIH }\end{array}$ & $\begin{array}{l}\text { En trevista para evaluar } \\
\text { la aceptabilidad del } \\
\text { video y su influencia en } \\
\text { la detección del VIH. }\end{array}$ & $\begin{array}{l}\text { 90\% de los médicos reportó que el } \\
\text { video les gustó mucho y } \\
\text { consideran que el video es viable y } \\
\text { pertinente para usarse con los } \\
\text { pacientes. }\end{array}$ \\
\hline 2 & $\begin{array}{l}\text { Bauermeist } \\
\text { er et al. } \\
2019\end{array}$ & $\begin{array}{l}155 \text { Jóvenes } \\
\text { gais, } \\
\text { bisexuales y } \\
\text { hombres que } \\
\text { tienen sexo } \\
\text { con hombres. } \\
\text { Edad } \\
\text { promedio: } 21 \\
\text { años }\end{array}$ & $\begin{array}{l}\text { Intervención en linea } \\
\text { basada en un marco } \\
\text { de toma de decisiones } \\
\text { cognitivo-emocional } \\
\text { para reducir } \\
\text { conductas sexuales de } \\
\text { riesgo. El grupo } \\
\text { control, solo recibió } \\
\text { información sobre los } \\
\text { contenidos de la } \\
\text { intervención en línea. }\end{array}$ & $\begin{array}{l}\text { Aceptabilidad: un } \\
\text { reactivo en formato tipo } \\
\text { likert para evaluar su } \\
\text { satisfacción y } \\
\text { recomendación de la } \\
\text { intervención. } \\
\text { Usabilidad: a través de } \\
\text { System Quality } \\
\text { subscale of the } \\
\text { Information Systems } \\
\text { Success Model con seis } \\
\text { reactivos sobre la } \\
\text { facilidad de uso de la } \\
\text { intervención. Utilidad: } \\
\text { a través de la Perceived } \\
\text { Usefulness subscale of } \\
\text { the Information Systems } \\
\text { Success Model que } \\
\text { constade } 7 \text { reactivos en } \\
\text { formato tipolikert sobre } \\
\text { qué tantoles sirve en su } \\
\text { conducta sexual. }\end{array}$ & $\begin{array}{l}\text { Los participantes del grupo } \\
\text { experimental informaron mayor } \\
\text { satisfacción y disposición para } \\
\text { recomendar la intervención a } \\
\text { amigos. Tendrian más } \\
\text { probabilidades de continuar } \\
\text { usandolaintervención si estuviera } \\
\text { disponible, percibieron mayor } \\
\text { utilidad de la intervención y tenian } \\
\text { más probabilidades de informar } \\
\text { que la intervención facilitó llevar } \\
\text { una vida más saludable que los del } \\
\text { grupo control. }\end{array}$ \\
\hline 3 & $\begin{array}{l}\text { Bond \& } \\
\text { Ramos } \\
(2019)\end{array}$ & $\begin{array}{l}116 \text { mujeres. } \\
\text { Edad } \\
\text { promedio: } 34 \\
\text { años }\end{array}$ & $\begin{array}{l}\text { Fomentar la adopción } \\
\text { de PEP y PrEP a través } \\
\text { de un video de salud } \\
\text { electrónica }\end{array}$ & $\begin{array}{l}\text { Explorar la viabilidad, } \\
\text { aceptabilidad y } \\
\text { preferencia de un video } \\
\text { sobre el uso de PEP y } \\
\text { PrEP para mujeres y } \\
\text { recomendación del } \\
\text { video. No indica } \\
\text { número de reactivos, } \\
\text { pero fue en formato tipo } \\
\text { likert. }\end{array}$ & $\begin{array}{l}\text { El } 89 \% \text { de los participantes } \\
\text { calificaron el video como bueno o } \\
\text { excelente. Los motivos para } \\
\text { recomendar el video incluian que } \\
\text { era educativo, entretenido y } \\
\text { adecuado para mujeres. }\end{array}$ \\
\hline 4 & $\begin{array}{l}\text { Bonett et } \\
\text { al. }(2018)\end{array}$ & $\begin{array}{l}86 \text { hombres } \\
\text { jóvenes que } \\
\text { tienen sexo } \\
\text { con hombres }\end{array}$ & $\begin{array}{l}\text { Página web con } \\
\text { información y } \\
\text { motivación para } \\
\text { realizarse pruebas de } \\
\text { detección de VIH. }\end{array}$ & $\begin{array}{l}\text { Número de clics en } \\
\text { cada una de las } \\
\text { secciones de la página } \\
\text { Web y su compromiso } \\
\text { a través del tiempo que } \\
\text { los participantes }\end{array}$ & $\begin{array}{l}\text { Los participantes hicieron clic en } \\
\text { un total de } 10.28 \text { funciones en } \\
\text { cada sección de la intervención; el } \\
\text { tiempo promedio dedicado fue de } \\
322.67 \text { segundos. El análisis de } \\
\text { datos presentados ayuda a }\end{array}$ \\
\hline
\end{tabular}


Experiencias de al alcance que tienen sobre la intervención con HSH en la prevención del VIH, y que hayan realizado actividades de divulgación y servicios en linea. instituciones respecto al. (2019) (Promedio de experiencia clinica: 14 años) y 20 jóvenes (Edad promedio: 19 años).

Dietrich et 50 mujeres al. (2020) inscritas en la Red de ensayos de vacunas Edad promedio: 24 años al. (2020) que tienen
sexo hombres

con
promover la salud sexual y la prevención del VIH entre adolescentes.

Aplicación móvil para prevenir el consumo de drogas y reducir ITS/VIH para mejorar la comunicación entre médicos y jóvenes.

Aplicación móvil para un autorregistro sobre conductas sexuales de riesgo
Cordova et 6 médicos contra el VIH. Questionnaire-8. principales (1) facilitadores como intervención (Edad promedio: 37 años) y $118 \mathrm{al}$ grupo experimental (Edad promedio: 38 años).

10 Lopez et al. 39 mujeres (2020) jóvenes de reducir el riesgo de dedicaron a cada una de las secciones y el tiempo total dedicado a la in tervención.

Entrevista por teléfono para explorar sus experiencias perspectivas sobre los servicios de atención.

Client Satisfaction

Ocho reactivos en formato tipo likert para evaluar la viabilidad, aceptabilidad $\quad \mathrm{y}$ satisfacción de la intervención. Una entrevista con preguntas abiertas para profundizar en sus razones.

Preguntas abiertas sobre las barreras y facilitadores para completar intervención. la

Se identificaron cinco temas el uso del móvil (2) barreras relacionadas con el tiempo y la pareja, (3) poder de los incentivos, (4) sesgo de respuesta al proporcionar información sensible, y (5) recomendaciones para mejorar la intervención.

Usabilidad: se utilizó la Health Information Technology Usability Evaluation Scale (Health-ITUES) y el Post-Study System Usability

Questionnaire,

Aceptabilidad:

entrevistas personales sobre lo aprendido, sobre la percepción de la intervención, y recomendaciones de mejora.

Grupos focales para recopilar comentarios sobre la intervención, aceptabilidad desafios y facilitadores para su uso.

comprender cómo los usuarios interactúan con la intervención, esto es relevante dado que la presentación de informes y análisis de datos es un área de investigación poco explorada. Se tienen indicadores diferentes para evaluar su utilidad.

Los hallazgos indican que aunque todavia hay cierta incertidumbre sobre cómo realizar actividades en herramienta vital para la prevención del VIH y las ITS. Mencionan ventajas del alcance en ceso instantáneo a los servicios, y poblaciones de HSH. Las calidad, presupuesto y problemas de capacidad del personal para mejores prácticas y parámetros de evaluación para la divulgación en linea.

Más del $85 \%$ considera que la es viable, y su alta tanto en se tuvo una valta viabilidad aceptabilidad de la intervención entre los jóvenes y los médicos.

Los participantes informaron que la aplicación estaba libre de de usar y útil, con un enfoque atractivo que aumentó la aceptabilidad. Las áreas de oportunidad están en términos de incluir contenido educativo más avanzado, escenarios para jóvenes con más experiencia sexual y función de búsqueda para aumentar la accesibilidad de contenidos clave.

La mayoría de los participantes informaron una experiencia positiva con el programa, más del 93\% informó que era fácil o muy fácil de usar, mencionaron que la información era adecuada y que tuvieron la suficiente privacidad durante las sesiones, y su mayoría mencionó la preferencia por el asesoramiento por computadora en lugar de hacerlo cara a cara.

Usabilidad Se encon tró una buena aceptación aceptabilidad: $\quad$ Se y usabilidad; indicaron que el divulgación en línea es una lineay las ventajas del anonimato, problemas funcionales, que es fácil 
zonas sada virtual (formato promedio: 21 de videoconferencias). años

Madkins et 445 hombres al. (2019) que tienen sexo con hombres. Edad promedio: 24 años (2020) y mujeres heterosexuale s. Edad promedio: 23 años. 30 asignados al grupo control y 30 al grupo experimental. Edad promedio: 12 años.

Shrestha et 40 al. (2020)
40
dependientes de opioides que usan PrEP (profilaxis previa a la
Intervención en línea para prevenir el VIH para $\mathrm{HSH}$.

ion adaptada por computadora para promover el uso del condón en relaciones sexuales.

evaluó a partir de tres reactivos en formato tipo likert sobre la recomendación del programa a amigos, contenido apropiado y atractivo, y si el formato de la videoconferencia era fácil de usar.

Aceptabilidad

tolerabilidad a partir de la adapted version of the Abbreviated Acceptability Rating

Profile que consta de 15 preguntas abiertas y 15 en formato tipo likert, a través de una clasificación de estrellas para evaluar el contenido in teractivo y video de cada módulo de intervención, asî como el tiempo dedicado a la intervención.

Aceptabilidad de la intervención. reactivos para evaluar si les gustó la intervención, si mantuvo su atención, cuánta información leyeron o escucharon, si se ajusta a sus necesidades, si sintieron que fue diseñado para ellos, qué tan fácil o difícil encontraron el programa, si regresarian para otra sesión, y si recomendaría el programa a un amigo. Viabilidad (datos de inscripción, retención y tasa de finalización. Aceptabilidad, en un formato tipo likert debian indicar qué tanto les gusto participar en la intervención. Además de grupos focales para identificar lo que más le gustó, lo que no le gustó y sugerencias para mejorar la in tervención. Viabilidad. A través de una seria de preguntas sobre el proceso de reclutamiento,

inscripción, deserción, seguridad de los participantes y devolución de teléfonos y experiencia del juego.

Intervención a través de mensajería de texto para la prevención del VIH y uso del PrEP.

Viabilidad: A través de indicadores de proceso (número de personas examinadas, inscritas y retenidas durante todo el período de estudio). Aceptabilidad: 10 ítems contenido era atractivo y fácil de usar y que recomendarian el programa a sus amigos. Las participantes indicaron que pudieron entablar relaciones con otras participantes a través de la plataforma y pudieron aprender habilidades útiles.

Los participantes calificaron muy bien el contenido, es decir que era útil y atractivo, pasaron aproximadamente 65 minutos en la intervención. El formato de presentación les gustó, pero el tiempo dedicado a la in tervención lo consideran largo. Una de las áreas de oportunidad se relaciona con la batería de preguntas que lo consideraron bastante largo.

Los indicadores más altos de aceptabilidad se obtuvieron en volver para otra sesión, recomendación a un amigo, leer o escuchar la información, y encontrar el programa fácil de utilizar. El indicador con la calificación más baja fue que no consideraban que la información fuese diseñada específicamente para ellos. Las mujeres tenían más probabilidades que los hombres de encontrar aceptable la intervención.

Al $82.3 \%$ les gustó o les gustó mucho participar en la intervención. Fue aceptable para los participantes, consideran que la intervención fue útil y proporcionó información de apoyo. Con respecto a las áreas de oportunidad informaron que la encuesta era demasiado larga o redundante, también tenían la sensación de que los men sajes no eran tan frecuentes.

Los resultados indican que es factible y seguro probar una intervención de preven ción del VIH basada en teléfonos inteligentes para jóvenes adolescentes. Los participantes indicaron que es viable la intervención.

La viabilidad del servicio de mensajes de texto fue alta, según la evaluación de la disposición de los participantes para recibir mensajes de texto, retención y entrega exitosa de mensajes de texto. También mostraron que 
exposición) y que son $\mathrm{VIH}$ negativo.

Edad

promedio: 44 años

16

Suryavans 30 mujeres. hi et al. 15 mujeres (2020)

17

Tanner et 18 hombres al. (2020) que tienen sexo con hombres y mujeres transgénero con VIH.

Edad promedio: 25 años

promedio: 23 años.

Trabajadoras extensión:

Edad promedio: 39 de una versión adaptada de Profile.32 en formato tipo likert. Adicionalmente se realizaron entrevistas semiestructuradas para obtener comentarios adicionales sobre la intervención (facilidad de comprensión, problemas, experiencia general, satisfacción y probabilidad de seguir usando la intervención).

Intervención basada en tecnología (móvil) para ayudar a los prestadores de servicios de prevención de $\mathrm{VIH}$ de la transmisión materno infantil

Intervención que utiliza plataformas de redes sociales diseñadas para la prevención del VIH.
Viabilidad intervención. Se realizaron entrevistas en profundidad.

Mujeres embarazadas: Percepciones sobre la eficacia y utilidad de la in tervención.

Prestadoras de servicio: Experiencia y percepciones de la intervención.

Entrevistas sobre las experiencias con la in tervención, beneficios de la participación, y recomendaciones de mejora. aceptabilidad de la estaban satisfechos y percibieron el uso de los mensajes de texto como valiosos y aceptables.
Mujeres embarazadas: Informaron que los videos de prevención eran esenciales para proporcionar información fácil de entender, mencionaron que la intervención aumen tó su confianza, motivación. Trabajadoras de extensión: Les brindó herramientas para brindar un apoyo eficaz a sus clientes y mejoró su estilo de trabajo. De manera general la intervención resultó factible, benéfica y bien aceptada.

Las recomendaciones de los entrevistados se centran en la logística, el contenido y las formas en que la intervención podría adaptarse para llegar a una audiencia más amplia. Se resalta el valor de utilizar plataformas de redes sociales sobre otros métodos de comunicación tradicional, resaltan la importancia de que los mensajes puedan ser bidireccionales y no automatizados, ampliar la intervención e incorporar componentes interactivos.

Nota de la Tabla: Se detallan los diez artículos seleccionados.

Groso modo se observa en los estudios reportados que la mayor parte de las intervenciones tienen un grupo para evaluar la aceptabilidad y viabilidad de la intervención (82\%), únicamente tres investigaciones lo hacen considerando grupo experimentaly grupo control (Bauermeister et al., 2019) (Kurth et al., 2019) (Sabben et al., 2019).

En la tabla 2 se presenta el objetivo que tuvo cada intervención, así como la población que se atendió, siendo el principal objetivo reducir conductas sexuales de riesgo, como el uso del condón, únicamente la investigación de Patel et al. (2020) además de lo anterior, también 
consideró dentro de sus metas el que las personas se realizaran pruebas de detección de VIH; también en esta misma tabla se observa que la mayor parte de la población en la que se trabaja es hombres que tienen relaciones sexuales con hombres (HSH).

\section{Tabla 2}

Estudios de evaluación sobre aceptabilidad y viabilidad de intervenciones mediadas por tecnología en la prevención del VIH de 2018 a 2020.

\begin{tabular}{|c|c|c|c|}
\hline & Estudios & $\mathbf{n}$ & $\%$ \\
\hline \multicolumn{4}{|l|}{ Objetivo } \\
\hline $\begin{array}{l}\text { Reducir conductas sexuales de } \\
\text { riesgo }\end{array}$ & $\begin{array}{l}\text { Bauermeister et al. (2019); Brennan et al. (2018); Cordova et al. } \\
\text { (2019); Dietrich et a. (2020); Gannon et al. (2020); Kurth et al. (2019); } \\
\text { Lopez et al. (2020); Madkins et al. (2019); Noar et al. (2020); *Patel et } \\
\text { al. (2020); Sabben et al. (2019); Tanner et al. (2020). }\end{array}$ & 12 & $67 \%$ \\
\hline Pruebas de detección de VIH & Arya et al. (2019); Bonett et al. (2018); *Patel et al. (2020). & 3 & $17 \%$ \\
\hline Uso de PEP y PrEP & Bond \& Ramos (2019); Shrestha et al. (2020). & 2 & $11 \%$ \\
\hline \multirow[t]{2}{*}{$\begin{array}{l}\text { Reducir infección materno } \\
\text { infantil }\end{array}$} & Suryavanshi et al. (2020). & 1 & $6 \%$ \\
\hline & & 18 & $100 \%$ \\
\hline HSH & $\begin{array}{l}\text { Bonett et al. (2018); Gannon et al. (2020); Madkins et al. (2019); Patel } \\
\text { et al. (2020); Tanner et al. (2020). }\end{array}$ & 5 & 29 \\
\hline Mujeres & $\begin{array}{l}\text { Bond \& Ramos (2019); Dietrich et al. (2020); Lopez et al. (2020); } \\
\text { Suryavanshi et al. (2020). }\end{array}$ & 4 & 24 \\
\hline Profesionales de la salud & Arya et al. (2019); Brennan et al. (2018); Cordova et al. (2019). & 3 & 18 \\
\hline Población en general & Noar et al. (2020); Sabben et al. (2019). & 2 & 12 \\
\hline Comunidad LGBT & Bauermeister et al. (2019). & 1 & 6 \\
\hline & Kurth et al. (2019). & 1 & 6 \\
\hline \multirow[t]{2}{*}{ Usuarios de PrEP } & Shrestha et al. (2020). & 1 & 6 \\
\hline & & 17 & 100 \\
\hline
\end{tabular}

Nota: *Estudio que tuvo dos objetivos.

Se explicitan los estudios de evaluación específicos de la aceptabilidad y viabilidad de intervenciones mediadas por tecnología en la prevención del VIH.

En la tabla 3 se hace clasificación de los artículos en función de la estrategia de recolección de la información para evaluar la aceptabilidad y la viabilidad de la intervención mediada por tecnología, en relación con el medio tecnológico utilizado en la intervención; por un lado, se observa que la intervención en línea y las aplicaciones móviles son las más utilizadas en los últimos años, en las cuales se comparte como característica principal el uso de internet; por otro lado, se observa que 
la forma más común para evaluar la viabilidad y la aceptabilidad de las intervenciones es mediante entrevistas individuales e instrumentos en formato tipo Likert, aunque cabe señalar que para las intervenciones en línea se utilizan en mayor medida escalas, y para las aplicaciones móviles, entrevistas individuales.

También es notorio que cuatro investigaciones hacen una combinación de estrategias de recolección de la información, tanto cuantitativas, como cualitativas para recolectar la información, siendo esto más común en las intervenciones con aplicación móvil. Si bien las estrategias de grupo focales y el número de clics que dan a un video o página Web se utilizan en menor medida, son posibilidades que tienen los investigadores para poder evaluar la aceptabilidad y viabilidad de la intervención.

\section{Tabla 3}

Comparación de la estrategia de recolección de la información para evaluar aceptabilidad y viabilidad con el medio tecnológico utilizado en la intervención para la prevención del VIH de 2018 a 2020.

\begin{tabular}{|c|c|c|c|c|c|}
\hline & $\begin{array}{l}\text { Entrevistas } \\
\text { individuales }\end{array}$ & Escala likert & Grupos focales & Número de clics & $\begin{array}{l}\text { Porcentaje } \\
\text { M edio de } \\
\text { intervención }\end{array}$ \\
\hline $\begin{array}{l}\text { Intervención } \\
\text { en linea }\end{array}$ & $\begin{array}{l}\text { Brennan et al. } \\
(2018) \text {. }\end{array}$ & $\begin{array}{l}\text { Bauermeister et al. } \\
\text { (2019); Lopez et al. } \\
(2020) \text {; Madkins et } \\
\text { al. (2019); Noar et } \\
\text { al. (2020); *Patel et } \\
\text { al. (2020). }\end{array}$ & $\begin{array}{l}\text { Kurth et al. (2019); } \\
{ }^{*} \text { Patel et al. (2020). }\end{array}$ & & $7(41 \%)$ \\
\hline $\begin{array}{l}\text { Aplicación } \\
\text { móvil }\end{array}$ & $\begin{array}{l}\text { *Cordova et al. } \\
(2019) ; \text { Dietrich et } \\
\text { al. }(2020) ;{ }^{*} \text { Gannon } \\
\text { et al. }(2020) ; \\
\text { Sabben et al. } \\
\text { (2019); }{ }^{\circ} \text { Shrestha et } \\
\text { al. (2020); } \\
\text { Suryavanshi et al. } \\
(2020) \text {. }\end{array}$ & $\begin{array}{l}\text { *Cordova et al. } \\
(2019) ;{ }^{*} \text { Gannon et } \\
\text { al. (2020); } \\
\text { *Shrestha et al. } \\
(2020) \text {. }\end{array}$ & & & $6(35 \%)$ \\
\hline Video & Arya et al. (2019). & & & $\begin{array}{l}\text { Bond \& Ramos } \\
\text { (2019). }\end{array}$ & $2(12 \%)$ \\
\hline $\begin{array}{l}\text { Página W eb } \\
\text { Redes } \\
\text { sociales }\end{array}$ & Tanner et al. (2020). & & & Bonett et al. (2018). & $\begin{array}{l}1(6 \%) \\
1(6 \%)\end{array}$ \\
\hline $\begin{array}{l}\text { Porcentaje } \\
\text { Forma de } \\
\text { evaluación }\end{array}$ & $9(43 \%)$ & $8(37 \%)$ & $2(10 \%)$ & $2(10 \%)$ & $100 \%$ \\
\hline
\end{tabular}

Nota: *Estudios con dos formas de evaluación de aceptabilidad y viabilidad de la intervención. 


\section{Discusión}

El objetivo de la presente revisión sistemática fue analizar publicaciones centradas en la evaluación de la aceptabilidad y viabilidad de intervenciones mediadas por tecnología orientadas en la prevención del VIH, para ello se buscó en tres bases de datos especializadas las publicaciones entre el 2018 y el 2020. Para este análisis se tomó en cuenta la búsqueda de artículos especializadas en tres bases de datos, con base en el cribaje realizado se obtuvo un total de 17 artículos, los cuales fueron revisados y analizados a detalle.

De manera general se observa que los estudios reportados, en su mayoría están centrados en la reducción de conductas sexuales de riesgo, esto probablemente se deba a que como se ha reportado en los últimos años, más del 90\% de las infecciones porVIH es porvía sexual (ONUSIDA, 2021), de tal manera que es una de las metas que se tiene en diferentes organismos internacionales, en las que se busca que las personas sean consistentes en el uso del condón, que lo usen de manera correcta, que reduzcan el número de parejas sexuales, entre otras (Evans et al., 2020).

Dentro de estos mismos objetivos que tienen las intervenciones se encuentra el que las personas se realicen pruebas de detección de VIH, de tal manera que conozcan su estado serológico y más aún si han tenido conductas sexuales de riesgo, ya que como se ha observado no todas las personas tienen este hábito (Shrestha et al., 2020); también en los últimos años ha cobrado gran relevancia el uso de PrEP como una alternativa en la que las personas pueden tomar una pastilla cuando han tenido alguna conducta de riesgo sexual para prevenir la infección por VIH (Camlin et al., 2020).

Si bien todas las personas están en riesgo de infectarse por VIH cuando tienen relaciones sexuales, hay ciertos contextos que hacen más probable 
que haya una infección, entre ellos se encuentran los HSH, y tal y como se encontró en la presente revisión, la mayor parte de la investigación se centra en este sector (Bonett et al., 2018) (Gannon et al. 2020) (Madkins et al., 2019) (Patel et al., 2020) (Tanner et al., 2020); seguido de mujeres que también son un grupo vulnerable para la infección, sobre todo por cuestiones de inequidad (Bond \& Ramos, 2019) (Dietrich et al., 2020) (Lopez et al., 2020) (Suryavanshi et al., 2020).

Cabe señalar que, en la población de atención, se encontró que en tercer lugar se encuentra el trabajo con profesionales de la salud, lo cual es fundamental y necesario, dado que ellos son quienes brindan un servicio de atención de VIH, y se ha observado una relación entre esta atención y que las personas respondan adecuadamente a la intervención (Ebrahimi et al., 2020). De igual forma en menor medida, pero no menos importante se tiene como meta la atención de la población en general (Noar et al., 2020) (Sabben et al., 2019), la comunidad LGBT (Bauermeister et al., 2019), población con VIH (Kurth et al., 2019) y usuarios de PrEP (Shrestha et al.; 2020), lo cual muestra la necesidad de seguir desarrollando intervenciones en estos contextos, pero al mismo tiempo identificar en quién más se debe prestar atención y que también requieren de una intervención para promover la salud sexual y reducir los riesgos de infección por VIH.

Respecto al punto central del presente trabajo, fue identificar cuáles son las estrategias de recolección de la información para evaluar la aceptabilidad y la viabilidad de las intervenciones, pero al mismo tiempo hacer un cruce con el medio tecnológico empleado en la intervención, de tal manera de tener indicadores que permitan identificar cuáles serían las mejores opciones para hacer dicha evaluación. 
Se encontró que son las entrevistas individuales a través de preguntas abiertas el medio que más se utiliza, seguida de instrumentos en formato tipo likert; si bien, en dichas investigaciones se resalta la facilidad que tiene por ejemplo el uso de escalas para valorar su pertinencia (Bauermeister et al., 2019) (Lopez et al., 2020), es cierto, que las preguntas abiertas no solo permiten conocer si perciben adecuada o no la intervención, sino que además dan información sobre las razones de dicha respuesta e inclusive cuáles podrian ser las áreas de oportunidad para mejorar las intervenciones (Dietrich et al., 2020) (Suryavanshi et al., 2020). Siendo una combinación de ambas estrategias quienes algunos autores consideran necesario para tener mejores indicadores (Cordova et al., 2019) (Gannon et al., 2020) (Patel et al. 2020) (Shrestha et al., 2020).

De manera general en los 17 estudios se encontró que las personas evalúan de manera favorable las intervenciones mediadas por tecnología, permitiendo una mayor motivación para su empleo, se vuelve más atractivo para concluir la intervención, de tal manera que la presente investigación, muestra que las intervenciones mediadas por tecnologia son pertinentes y necesarias (Bond \& Ramos 2019) (Cordova et al., 2019) (Gannon et al., 2020).

Respecto a las áreas de oportunidad, se encuentra que es necesario que la intervención tome en cuenta a quién va dirigida, de tal manera que los ejemplos sean contextuales (Noar et al., 2020), que la intervención sea más interactiva (Tanner et al., 2020), y sobre el tiempo, ya sea respecto a la duración de la intervención o la extensión de los instrumentos de evaluación que responden antes y después de la intervención (Madkins et al., 2019) (Patel et al., 2020).

Si bien solo se encontró dos estudios que utilizan los grupos focales para evaluar la aceptabilidad y viabilidad de la intervención, es pertinente 
su uso sobre todo cuando se tienen varios participantes, e inclusive de acuerdo con Fernández-Sánchez et al. (2020), la evaluación sobre el estudio piloto de las intervenciones debe ser tal cual se pretende llevar a cabo, en donde se privilegia los diseños controlados aleatorizados (ZuritaCruz et al., 2018), y un ejemplo de que es posible hacerlo es la investigación de Kurth et al. (2019).

También, una nueva propuesta que se hace respecto a la evaluación de la aceptabilidad y viabilidad de la intervención es la realizada por Bond y Ramos (2019), y (Bonett et al., 2018), quienes proponer evaluar a través del número de clics que las personas hacen durante la intervención, en relación con el tiempo que están dentro de la intervención y el tiempo que les lleva terminarla; por lo cual se sugiere que en dicha evaluación se pueda hacer una combinación de entrevistas, cuestionarios y número de clics para comparar los resultados entre sí y poder tener un indicador de cuál podría ser más viable o si es necesario contar con todos ellos para su evaluación.

\section{Conclusiones}

La evaluación de las intervenciones mediadas por tecnologias, han cobrado gran auge en las últimas décadas, aunque cabe señalar que son más bien las intervenciones mediadas por internet las que están predominando en los últimos años, por lo cual es un aspecto importante para considerar cuando se establezca un protocolo de intervención para promover la salud sexual y reducir la infección por VIH.

Un punto importante en toda intervención y que es necesario evaluar es qué tan pertinente es llevar a cabo la in tervención y qué tan relevante es para la vida de las personas dichaintervención, por lo cual se requiere de su valoración por parte de los usuarios de tal manera que se pueda 
mejorar la intervención para lograr realmente un impacto en la vida de las personas.

En el caso particular de la prevención del VIH, es fundamental dado que está en juego la salud de las personas, y las intervenciones mediadas por internet pueden ser una opción viable y pertinente para lograrlo. 


\section{Referencias}

Anstee, S., Shepherd, J., Graham, C. A., Stone, N., Brown, K., Newby, K., \& Ingham, R. (2019). Evidence for behavioural interventions addressing condom use fit and feel issues to improve condom use: a systematic review. Sexual Health, 16(6), 539-547. https://doi.org/10.1071/SH19001

Arya, M., Marek, H. G., Marren, R. E., Hemmige, V., Street, R. L., \& Giordano, T. P. (2019). Development and evaluation of a physician-targeted video to promote HIV screening. Health Promotion Practice, 20(6), 922-931. https://doi.org/10.1177/1524839918783742

Bauermeister, J. A., Tingler, R. C., Demers, M., Connochie, D., Gillard, G., Shaver, J., Chavanduka, T., \& Harper, G. W. (2019). Acceptability and preliminary efficacy of an online HIV prevention intervention for single young men who have sex with men seeking partners online: The myDEx Project. Aids and Behavior, 23(11), 3064-3077. https://doi.org/10.1007/s10461$\underline{019-02426-7}$

Bond, K. T., \& Ramos, S. R. (2019). Utilization of an animated electronic health video to increase knowledge of post- and pre-exposure prophylaxis for HIV among African American Women: Nationwide cross-sectional survey. JMIR Formative Re search, 3(2), e 9995. https://doi.org/10.2196/formative.9995

Bonett, S., Connochie, D., Golinkoff, J. M., Horvath, K. J., \& Bauermeister, J. A. (2018). Paradata analysis of an ehealth HIV testing intervention for young men who have sex with men. Aids Education and Prevention, 30(5), 434-447. https://doi.org/10.1521/aeap.2018.30.5.434

Brennan, D. J., Souleymanov, R., Lachowsky, N., Betancourt, G., Pugh, D., \& McEwen, O. (2018). Providing online-based sexual health outreach to gay, bisexual, and queer men in Ontario, Canada: Qualitative interviews with multisectoral frontline service providers and $\mathrm{m}$ anagers. Aids Patient Care and Stds, 32(7), 282-287. https://doi.org/10.1089/a pc.2018.0027

Camlin, C. S., Koss, C. A., Getahun, M., Owino, L., Itiakorit, H., Akatukwasa, C., Maeri, I., Bakanoma, R., Onyango, A., Atwine, F., Ayieko, J., Kabami, J., Mwangwa, F., Atukunda, M., Owaraganise, A., Kwarisiima, D., Sang, N., Bukusi, E. A., Kamya, M. R., Petersen, M. L., Cohen, C. R., Charlebois, E. D., \& Havlir, D. V. (2020). Understanding demand for PrEP and early experiences of PrEP use among young adults in rural Kenya and Uganda: A qualitative study. AIDS and Behavior, 24(7), 2149-2162. https://doi.org/10.1007/s10461-020-02780-x

Cordova, D., Mendoza, F., Muñoz-Velázquez, J., Street, K., Bauermeister, J. A., Fessler, K., Adelman, N., Youth Leadership Council, Neilands, T. B., \& Boyer, C. B. (2019). A multilevel mHealth drug abuse and STI/HIV preventive intervention for clinic settings in the United States: A feasibility and acce ptability study. PLoS ONE, 14(8), 1-24.

https://doi.org/10.1371/journal. pone.0221508

Dietrich, J. J., Hornschuh, S., Khunwane, M., Makhale, L. M., Otwombe, K., Morgan, C., Huang, Y., Lemos, M., Lazarus, E., Kublin, J. G., Gray, G. E., Laher, F., \& Andrasik, M. (2020). A mixed methods investigation of implementation barriers and facilitators to a daily mobile phone sexual risk assessment for young women in Sowe to, South Africa. PLoS ONE, 15(4), Article e0231086. https://doi.org/10.1371/journal. pone.0231086

Ebrahimi, F., Sindarreh, S., \& Nasirian, M. (2020). Attitudes and Practice of Health Care Workers about Human Immunode ficiency Virus in Isfahan, Iran. Iranian Journal of Nursing \& Midwifery Research, 25(2), 111-116. https://doi.org/10.4103/ijnmr.IJNMR_67_19

Evans, W. D., Ulasevich, A., Hatheway, M., \& Deperthes, B. (2020). Systematic Review of PeerReviewed Literature on Global Condom Promotion Programs. International Journal of Environmental Re search and Public Health, 17(7), 2262. https://www.mdpi.com/1660$\underline{4601 / 17 / 7 / 2262}$ 
Fan, X., She, R., Liu, C., Zhong, H., Lau, J. T. F., Hao, C., Li, J., Hao, Y., Li, L., \& Gu, J. (2020). Evaluation of smartphone APP-based case-management services among antiretroviral treatment-naïve HIV-positive men who have sex with men: A randomized controlled trial protocol. BMC Public Health, 20(1), 1-13. https://doi.org/10.1186/s12889-020-8171-5

Fernández-Sánchez, H., Guzmán-Facundo, F. R., Herrera-Medina, D., \& Sidani, S. (2020). Importancia del estudio piloto en un proyecto de intervención. Index De Enfermeria, (Preedición), e 12860. http:// ciberindex.com/c/ie/e 12860

Gannon, B., Davis, R., Kuhns, L. M., Rodriguez, R. G., Garofalo, R., \& Schnall, R. (2020). A mobile sexual health app on empowerment, education, and prevention for young adult men (MyPEEPS mobile): Acceptability and usability evaluation. Journal of Medical Internet Research, 22(4), e 17901. https://doi.org/10.2196/17901

Hightow-Weidman, L. B., LeGrand, S., Muessig, K. E., Simmons, R. A., Soni, K., Choi, S. K., Kirschke-Schwartz, H., \& Egger, J. R. (2019). A randomized trial of an online risk reduction intervention for young black MSM. Aids and Behavior, 23(5), 1166-1177.

https://doi.org/10.1007/s10461-018-2289-9

Hutton, B., Catalá-López, F., \& Moher, D. (2016). La extensión de la declaración PRISMA para revisiones sistemáticas que incorporan metaanálisis en red: PRISMA-NMA. Medicina clínica, 147(6), 262-266. https://doi.org/10.1016/j.medcli.2016.02.025

Knight, R., Karamouzian, M., Salway, T., Gilbert, M., \& Shoveller, J. (2017). Online interventions to address HIV and other sexually transmitted and blood-borne infections among young gay, bisexual and other men who have sex with men: A systematic review. Journal of the International AIDS Society, 20(3), e25017. https://doi.org/10.1002/jia2.25017

Kurth, A. E., Sidle, J. E., Chhun, N., Lizcano, J. A., Macharia, S. M., Garcia, M. M., Mwangi, A., Keter, A., \& Siika, A. M. (2019). Computer-based counseling program (Care+ Kenya) to promote prevention and HIV health for people living with HIV/AIDS: A randomized controlled trial. Aids Education and Prevention, 31(5), 395-406.

https://doi.org/10.1521/aeap.2019.31.5.395

Lopez, C., Gilmore, A. K., Moreland, A., Danielson, C. K., \& Acierno, R. (2020). Meeting kids where they are at-a substance use and sexual risk prevention program via telemedicine for african american girls: Usability and acceptability study. Journal of Medical Internet Research, 22(8), e 16725. https:/ / doi.org/10.2196/16725

Madkins, K., Moskowitz, D. A., Moran, K., Dellucci, T. V., \& Mustanski, B. (2019). Measuring acceptability and engagement of the keepit up! Internet-based HIV prevention randomized controlled trial for young men who have sex with men. Aids Education and Prevention, 31(4), 287-305. https://doi.org/10.1521/aeap.2019.31.4.287

Mustanski, B., Parsons, J. T., Sullivan, P. S., Madkins, K., Rosenberg, E., \& Swann, G. (2018). Biomedical and behavioral outcomes of keepit up!: An eHealth HIV prevention program RCT. American Journal of Preventive Medicine, 55(2), 151-158.

https://doi.org/10.1016/i.ame pre.2018.04.026

Noar, S. M., Willoughby, J. F., Crosby, R., Webb, E. M., Van Stee, S. K., Feist-Price, S., \& Davis, E. (2020). Acceptability of a computer-tailored safer sex intervention for heterosexually active African Americans attending an STI clinic. Journal of Primary Prevention, 41(3), 211-227. https://doi.org/10.1007/s10935-020-00585-1

Ogrinc, G., Davies, L., Goodman, D., Batalden, P., Davidoff, F., \& Stevens, D. (2015). SQUIRE 2.0 (Standards for Quality Improvement Reporting Excellence): Revised publication guidelines from a detailed consensus process. The Journal of Continuing Education in Nursing, 46(11), 501-507. https://doi.org/10.3928/00220124-20151020-02 
ONUSIDA (2021). Hoja informativa 2021. Estimaciones epidemiológicas preliminares de ONUSIDA para 2021.

https://www.unaids.org/sites/default/files/media_asset/UNAIDS_FactSheet_es.pdf

Pate1, V. V., Rawat, S., Dange, A., Lelutiu-Weinberger, C., \& Golub, S. A. (2020). An internet-based, peer-delivered messaging intervention for HIV testing and condom use among men who have sex with men in India (CHALO!): Pilot randomized comparative trial. JMIR Public Health and Surveillance, 6(2), e 16494. https:/ / doi.org/10.2196/16494

Refugio, O. N., Kimble, M. M., Silva, C. L., Lykens, J. E., Bannister, C., \& Klausner, J. D. (2019). Brief report: PrEPTECH: A telehealth-basedinitiation program for HIV pre-exposure prophylaxis in young men of color who have sex with men. A pilot study of feasibility. JAIDS Journal of Acquired Immune Deficiency Syndromes, 80(1). 40-45. https://doi.org/10.1097/QAI.0000000000001873

Sabben, G., Akelo, V., Mudhune, V., Ondeng'e, K., Ndivo, R., Stephenson, R., \& Winske1l, K. (2019). A smartphone game to prevent HIV among young Africans: Protocol for a randomized pilot study of a mobile intervention. JMIR Research Protocols, 8(3), e 11209. https://doi.org/10.2196/11209

Shrestha, R., Altice, F. L., DiDomizio, E., Sibilio, B., Ranjit, Y. S., \& Copenhaver, M. M. (2020). Feasibility and acceptability of an mHealth-based approach as an HIV prevention strategy among people who use drugs on pre-exposure prophylaxis. Patient Preference $\&$ Adherence, 14, 107-118. https://doi.org/10.2147/PPA.S236794

Sullivan, P. S., Driggers, R., Stekler, J. D., Siegler, A., Goldenberg, T., McDougal, S. J., Caucutt, J., Jones, J., \& Stephenson, R. (2017). Usability and acceptability of a mobile comprehensive HIV prevention app for men who have sexwith men: A pilot study. JMIR Mhealth Uhealth, 5(3), e26. https://doi.org/10.2196/mhealth.7199

Suryavanshi, N., Kadam, A., Gupte, N., Hegde, A., Kanade, S., Sivalenka, S., Kumar, V. S., Gupta, A., Bollinger, R. C., Shankar, A., McKenzie-White, J., \& Mave, V. (2020). A mobile healthfacilitated behavioural intervention for community health workers improves exclusive breastfeeding and early infant HIV diagnosis in India: A cluster randomized trial. Journal of the International AIDS Society, 23(7), 1-9. https://doi.org/10.1002/jia2.25555

Suryavanshi, N., Kadam, A., Kanade, S., Gupte, N., Gupta, A., Bollinger, R., Mave, V., \& Shankar, A. (2020). Acceptability and feasibility of a behavioral and mobile health intervention (COMBIND) shown to increase uptake of prevention of mother to child transmission (PMTCT) care in India. BMC Public Health, 20(1), 752. https://doi.org/10.1186/s12889-020-08706-5

Tanner, A. E., Mann-Jackson, L., Song, E. Y., Alonzo, J., Schafer, K. R., Ware, S., Horridge, D. N., Garcia, J. M., Bell, J., Hall, E. A., Baker, L. S., \& Rhodes, S. D. (2020). Supporting health among young men who have sex with men and transgender women with HIV: Lessons learned from implementing the we Care intervention. Health Promotion Practice, 21(5), 755-763. https://doi.org/10.1177/1524839920936241

Zurita-Cruz, J., Márquez-González, H., Miranda-Novales, G., \& Villasís-Keever, M. (2018). Estudios experimentales: diseños de investigación para la evaluación de intervenciones en la clínica. Revista alergia México, 65(2), 178-186. https://doi.org/10.29262/ram.v65i2.376 